American Journal of Applied Sciences 4 (12): 977-986, 2007

ISSN 1546-9239

(C) 2007 Science Publications

\title{
Fault Detection and Isolation in Dynamic Systems using Statistical Local Approach and Hybrid Least Squares Algorithm
}

\author{
Belkacem ATHAMENA, Zina HOUHAMDI and Mohammed MUHAIRAT \\ Software Engineering Department, Faculty of Science and Information Technology,
} Al-Zaytoonah University, Jordan

\begin{abstract}
A fault detection and isolation (FDI) scheme for dynamic system proposed. This study deals with the design of discrete-time linear system using delta operator approach and the hybrid least squares (HLS) algorithm. A third residual generation based on statistical local approach and the derivative of the normalized residual on a small temporal window investigated. This new technique meets the desired FDI performance specifications by increasing the faults magnitude and decreasing the noise effects. Some simulation results were provided to evaluate the design.
\end{abstract}

Key words: Hybrid least squares, Fault detection and isolation, Residual, Fault detectability and isolatability, Local approach

\section{INTRODUCTION}

The increasing complexity of modern automated processes and the increasing demands for quality, reliability, availability, safety and cost efficiency require better safety management and supervision. Generally, the function of a supervisory control system is to detect and isolate faults in the system. The design of many modern fault detection and diagnosis system is based on the mathematical model of the plants. The model-based technique is where the actual behavior of the system is compared with the nominal fault free model that is driven by the same input. The result of this comparison leads to class of signal called residual. If the residual is not zero, then the system has faults, otherwise the system is normal.

In general, fault detection and diagnosis is a board and active area of research. There are a large volume of papers that deal with this subject, see, e.g., ${ }^{[1]}$ and references therein. In many applications the problem of FDI is a crucial issue that has been theoretically and experimentally investigated with different types of $\operatorname{approaches}^{[2]}$.

A novel residual generation method is proposed in this paper, whereas the residual generation based on the derivation of the normalized residual in a small temporal window is developed. The proposed residual offers favorable FDI properties, in which decreasing the noise effects and increasing the faults magnitude, therefore the FDI are improved especially in the case of the small change in the physical parameters of the system.
The purpose of the paper is to describe and analyze the FDI algorithm, based on the HLS parameter estimation technique using delta operator and the statistical local approach. The delta operator, which proved to be a convenient tool for examining the asymptotic behavior of discrete-time models of continuous-time systems as the sampling period converges to zero offers several advantages as compared to the common forward shift $q$ operator often leading to ill-conditioned processes ${ }^{[3]}$. It has been observed that for sufficiently small sampling periods the $\delta$ operator algorithms not only are much less sensitive to arithmetic round-off errors than their counterparts $q$-domain algorithms, but also ensure that the delta representation of a discrete-time system will converge to the corresponding continuous-time system as the sampling period tends to zero ${ }^{[4]}$. The main advantage of the statistical local approach is its ability in assessing the level of significance of discrepancies with respect to uncertainties. This approach has a wide scope of interest, since it can encompass all the types of FDI problems, for sensors, actuators and components faults.

\section{DELTA-DOMAIN MODELING AND STIMATION}

Delta model: The problem of obtaining a $\delta$-domain discrete-time model for a given continuous-time system has been addressed by many authors ${ }^{[4]}$. A reliable algorithm for derivation of these models of continuous-

\section{Corresponding Author: Belkacem ATHAMENA, Zina HOUHAMDI, Software Engineering Department, Faculty of} Science and Information Technology, Al-Zaytoonah University, Jordan 
time systems in a finite precision arithmetic can be found in ${ }^{[3]}$.

Consider a single-input single-output linear continuous-time system,

$A^{\prime}(s) y(t)=B^{\prime}(s) u(t)$

where $u(t), y(t)$ denoting the continuous-time input and output respectively, $A^{\prime}(s)$ and $B^{\prime}(s)$ are polynomials of order $n$ and $m, m<n$, respectively, i.e.,

$$
\begin{aligned}
& A^{\prime}(s)=s^{n}+a_{n-1}^{\prime} s^{n-1}+\cdots+a_{0}^{\prime} \\
& B^{\prime}(s)=b_{m}^{\prime} s^{m}+\cdots+b_{0}^{\prime}, b_{m}^{\prime} \neq 0
\end{aligned}
$$

Let $T$ be the sample period, the delta operator defined by $[1,11]$,

$\delta=\frac{q-1}{T}$

can serve as an alternative, which is known as mediation between the differential and the shift operators. It has been shown that the delta operator offers advantages over the shift operator, in terms of numerical robustness ${ }^{[4]}$.

Using the delta operator (3), an alternative discretetime representation based on the delta operator of the previous model (1) is given by,

$A(\delta) y(t)=B(\delta) u(t)$

Letting $T \rightarrow 0$, then $A(\delta) \rightarrow A^{\prime}(s)$ and $B(\delta) \rightarrow B^{\prime}(s)$, i.e.,

$\left\{\begin{array}{l}A(\delta)=\delta^{n}+a_{n-1} \delta^{n-1}+\cdots+a_{0} \\ B(\delta)=b_{m} \delta^{m}+b_{m-1} \delta^{m-1}+\cdots+b_{0}\end{array}\right.$

This implies that the delta representation converge to the continuous-time representation as the sampling rate increase.

Hybrid parameter estimation: In case when the derivatives of $y(t), u(t)$ in (4) are not available, we can use filtered derivatives in place of the raw derivatives. Since $\delta^{i} y(t)$ involves near differentiation of the data, incorporate a polynomial pre-filter $E(\delta)$,

$E(\delta)=\delta^{n}+e_{n-1} \delta^{n-1}+\cdots+e_{0}$

(6)

by writing,

$y(t)=\frac{(E(\delta)-A(\delta))}{E(\delta)} y(t)+\frac{B(\delta)}{E(\delta)} u(t)$

(7)

the choice of $E(\delta)$ conditions the bias, but also the convergence of the estimation ${ }^{[4]}$.
Note that since both $A(\delta)$ and $B(\delta)$ have unity leading coefficients only data up to time $t$ is required on the right-side of (7),

$y(t)=[E(\delta)-A(\delta)] y_{F}(t)+B(\delta) u_{F}(t)$

where,

$$
y_{F}(t)=\frac{y(t)}{E(\delta)}, \quad \quad u_{F}(t)=\frac{u(t)}{E(\delta)}
$$

(9)

Now define a parameter vector for (8) as,

$\theta=\left[\begin{array}{llllll}e_{n-1}-a_{n-1} & \cdots & e_{0}-a_{0} & b_{m} & \cdots & b_{0}\end{array}\right]^{T}$

where $\theta=\left[\begin{array}{lll}\theta_{1} & \cdots & \theta_{l}\end{array}\right]^{T}, \quad l=\operatorname{dim}(\theta)=n+m+1$. Then the linear regression model can be written as,

$$
y(t)=\varphi_{F}^{T}(t) \theta
$$

where,

$\varphi_{F}^{T}(t)=\left[\begin{array}{llllll}\delta^{n-1} y_{F}(t) & \cdots & y_{F}(t) & \delta^{m} u_{F}(t) & \cdots & u_{F}(t)\end{array}\right]$

(12)

where $\varphi_{F}(t)$ is the regression vector. We obtain a linear model with regard to the parameters by a transformation of the original data to the filtered data, where an analogue relation to the equation (4).

For know orders and delay, the problem consists of estimating the parameter vector $\theta$ in the linear regression model (13) from the available data. In this paper, the HLS algorithm with forgetting factors $\lambda$ has been chosen, because it best fits to the problem studied and because it is easy to implement. Estimation of the parameter $\theta$ may be found by minimizing the following sum of square of the equation error,

$J(\theta, N)=\sum_{t=1}^{N} \lambda^{N-t}\left[y(t)-\varphi_{F}^{T}(t) \theta\right]^{2}$

However, the parameter vector $\theta_{0}$ that satisfy $\varphi_{F}^{T}(t) \theta_{0}=0$, is not unique. To let the estimation unique, a parameter constraint $\theta^{T} \theta=1$ may be used. For the recursive estimation of the parameter, the HLS algorithm with adaptive forgetting factors $\lambda(t)$ is used which is given as follows ${ }^{[4,5,6]}$,

$$
\left\{\begin{array}{l}
\hat{\theta}(t+1)=\hat{\theta}(t)+\frac{T P(t) \varphi_{F}(t) \varepsilon(t)}{\lambda(t)+T \varphi_{F}^{T}(t) P(t) \varphi_{F}(t)} \\
P(t+1)=\frac{1}{\lambda(t)}\left[P(t)-T(T+\lambda(t)) \frac{P(t) \varphi_{F}(t) \varphi_{F}^{T}(t) P(t)}{\lambda(t)+T \varphi_{F}^{T}(t) P(t) \varphi_{F}(t)}\right]
\end{array}\right.
$$

where $\varepsilon(t)$ is prediction error defined as,

$\varepsilon(t)=y(t)-\varphi_{F}^{T}(t) \hat{\theta}(t)$

and the adaptive forgetting factors $\lambda(t)$ is defined as,

$\lambda(t)=\lambda_{0} \lambda(t-1)+(1-\lambda(0))$ 
The forgetting factor is used mainly to put higher weights on the more recent measurements so as to facilitate the convergence rate of the estimation of timevarying parameters.

\section{FDI SCHEME}

FDI problem: The problem of fault detection consists in making the decision on the presence or absence of faults in a monitored system. When no fault is present in the system, the system is said in its safe mode; otherwise it is in a faulty mode.

Generally speaking, faults in a dynamic system are often associated with malfunction of system components, which are reflected as changes in the system parameters. This situation can be modeled as abrupt or slowly developing parameter changes. In the above model representation (13), this type of faults can be modeled as:

$$
\theta= \begin{cases}\theta_{0} & t<t_{f}: \text { fault free } \\ \theta_{0}+\Delta \theta / \sqrt{N} & t \geq t_{f}: \text { with fault }\end{cases}
$$

where $\theta_{0}$ (identified with data from the safe system) and $\Delta \theta$ (same dimension as $\theta_{0}$, but with an arbitrary direction and a small magnitude) represent the nominal system parameters and the fault-induced parameter changes, respectively, and $t_{f}$ is the fault occurrence time. The normalization $1 / \sqrt{N}$ is necessary in order to get a non-trivial limit distribution.

To model the process dynamics and to generate the residual parameter it can be assumed that the equivalent input-output representation of the model (13) described as,

$$
g\left(\theta, u_{F}, y_{F}, \delta\right)=y(t)-\varphi_{F}^{T}(t) \theta
$$

where $g\left(\theta, u_{F}, y_{F}, \delta\right)$ is a vector of differential polynomials in $u_{F}, y_{F}$ and $\theta$.

Residuals generation: In the previous studies ${ }^{[7]}$, the parametric statistical approach distinguishes between two residuals ${ }^{[7]}$ : primary residual, which is a function of the model parameter $\theta$ and the observations $u_{F}, y_{F}$, and normalized residual, which is the mean of the normalized sum of this primary residual. Our contribution in this study is the generation of a third residual based on the derivative of the normalized residual on a small temporal window.

Due to the modeling uncertainty and measurement errors, some stochastic assumption should be introduced in order to take them into consideration. For linear systems, noises are usually assumed to be additive in model equations. For the sake of simplicity, we assume that the stochastic model of the system is, $g\left(\theta, u_{F}, y_{F}, \delta\right)=\eta_{F}$

where the noise $\eta_{F}$ is assumed be Gaussian.

For a system modeled by equation (11), assume that the parameter $\theta$ is locally identifiable at the nominal value $\theta_{0}$. Let $\theta$ be the actual parameter value for the system which generated the new data sample. The primary residual related to the identification of $\theta$ by minimizing the square of $g\left(\theta, u_{F}, y_{F}, \delta\right)$ writes as,

$$
\begin{aligned}
R\left(\theta, u_{F}, y_{F}, \delta\right) & =-\frac{1}{2} \frac{\partial}{\partial \theta}\left[(y(t)-g(\cdot))^{T} \cdot(y(t)-g(\cdot))\right] \\
& =-\varphi_{F}(t) \varphi_{F}^{T}(t) \theta(t)
\end{aligned}
$$

can be interpreted as an efficient score for model (18), (19), and is of interest for monitoring purposes. This is because changes in $\theta$ are reflected by changes in the mean value of $R\left(\theta, u_{F}, y_{F}, \delta\right)$.

For $N$-size data sample, the cumulative sum of quantities $(20), r_{N}(\theta)$, is given by ${ }^{[1,8]}$,

$$
\begin{aligned}
r_{N}(\theta) & =\frac{1}{\sqrt{N}} \sum_{t=1}^{N} R\left(\theta, u_{F}, y_{F}, \delta\right) \\
& =\frac{-1}{\sqrt{N}} \sum_{t=1}^{N} \varphi_{F}(t) \varphi_{F}^{T}(t) \theta(t)
\end{aligned}
$$

Technical arguments for the $\sqrt{N}$ factor can be found in $[2,9]$. Let $E_{\theta}$ be the expectation when the actual system parameter is $\theta$, we know that ${ }^{[7]} r_{N}(\theta)$ is defined as a normalized residual if,

$$
\left\{\begin{array}{l}
E_{\theta}\left[r_{N}(\theta)\right]=0 \text { for } \theta=\theta_{0} \\
E_{\theta}\left[r_{N}(\theta)\right] \neq 0 \text { for } \theta=\theta_{0}+\Delta \theta / \sqrt{N}
\end{array}\right.
$$

In this paper, we propose a third residual generation $D_{N}(\theta)$ based on the derivative of the normalized residual $r_{N}(\theta)$, which is estimated on the same temporal window $N$,

$$
D_{N}(\theta)=\frac{d}{d t}\left[r_{N}(\theta)\right]
$$

Using the delta operator (section 2), the residual $D_{N}(\theta)$ can be expressed as,

$$
D_{N}(\theta)=\frac{1}{T \sqrt{N}} \sum_{t=1}^{N}\left(\varphi_{F}(t) \varphi_{F}^{T}(t) \theta(t)-\varphi_{F}(t+1) \varphi_{F}^{T}(t+1) \theta(t+1)\right)(24)
$$

note that, the use of derivative of the means values on a small temporal window allows the filtering of noises, and at the same time, allows a fast resolution of all changes in residuals ${ }^{[10]}$. It is precisely this quantity 
$D_{N}(\theta)$ that is subsequently evaluated in order to perform FDI. Under some conditions, the residual $D_{N}(\theta)$ is asymptotically Gaussian distributed, and reflects a small fault by a change in its mean vector.

To decide whether $\theta=\theta_{0}$ holds true or not, or equivalently whether the residual $D_{N}(\theta)$ is significantly different from zero, requires the knowledge of the probability distribution of $D_{N}\left(\theta_{0}\right)$, which unfortunately is generally unknown. One manner to circumvent this difficulty is to assume close hypotheses,

$\left\{\begin{array}{l}(\text { safe }) H_{0}: \theta=\theta_{0} \\ (\text { fault }) H_{1}: \theta=\theta_{0}+\Delta \theta / \sqrt{N}\end{array}\right.$

where vector $\Delta \theta$ is unknown but fixed. Note that for large $N$, hypothesis $H_{1}$ corresponds to small deviations in $\theta$.

Let $\Sigma\left(\theta_{0}\right)=\lim _{N \rightarrow \infty} \mathrm{E}_{\theta}\left(D_{N} D_{N}^{T}\right)$ be the residual covariance matrix. Matrix $\Sigma$ captures the uncertainty in $D_{N}$ due to estimation errors: indeed the covariance matrix of the error in estimating $\theta_{0}$ is this $\Sigma\left(\theta_{0}\right)$ as well ${ }^{[7]}$.

Provided that $\Sigma\left(\theta_{0}\right)$ is positive definite, the residual $D_{N}$ in (23) is asymptotically Gaussian distributed with the same covariance matrix $\Sigma\left(\theta_{0}\right)$ under both $H_{0}$ and $H_{1}$. According to ${ }^{[7]}$, the residual $D_{N}(\theta)$ have the following properties,

$D_{N}\left(\theta_{0}\right) \rightarrow \begin{cases}\mathrm{N}\left(-M\left(\theta_{0}\right) \Delta \theta, \Sigma\left(\theta_{0}\right)\right) & \text { under } H_{0} \\ \mathrm{~N}\left(0, \Sigma\left(\theta_{0}\right)\right) & \text { under } H_{1}\end{cases}$

where $M\left(\theta_{0}\right)$ is the Jacobean matrix containing the sensitivities of the residual with respect to the model parameters,

$$
\begin{aligned}
M\left(\theta_{0}\right) & =\left.\frac{1}{\sqrt{N}} \sum_{t=1}^{N} \frac{\partial}{\partial \theta} D_{N}(\theta)\right|_{\theta=\theta_{0}} \\
& \left.=\frac{1}{T N} \sum_{t=1}^{N} \varphi_{F}(t) \varphi_{F}^{T}(t)-\varphi_{F}(t+1) \varphi_{F}^{T}(t+1)\right]
\end{aligned}
$$

As seen in (26), a deviation $\Delta \theta$ in the system parameter $\theta$ is reflected into a change in the mean value of residual $D_{N}$, which switches from zero to $M\left(\theta_{0}\right) \Delta \theta$ in case of small damage. Note that matrices $M\left(\theta_{0}\right)$ and $\Sigma\left(\theta_{0}\right)$ depend on neither the sample size $N$ nor the fault vector $\Delta \theta$ in hypothesis $H_{1}$. Thus they can be estimated prior to testing, using data on the safe system (exactly as the reference parameter $\theta_{0}$ ).
Fault detection and detectability: For deciding between $\Delta \theta=0$ and $\Delta \theta \neq 0$, the decision can be taken based on the optimum test statistics $\tau$, referred as the global test, is based on the generalized log-likelihood ratio. It can be shown ${ }^{[1,7]}$ that the GLR test of $H_{1}$ against $H_{0}$ can be written as,

$\tau=D_{N}^{T} \Sigma^{-1} M\left(M^{T} \Sigma^{-1} M\right)^{-1} M^{T} \Sigma^{-1} D_{N}$

which should be compared to a threshold. If the incidence matrix $M$ is an invertible matrix, then the global test $\tau$ can be reduces to,

$\tau=D_{N}^{T} \Sigma^{-1} D_{N}$

is asymptotically $\chi^{2}$-distributed, with a number of degrees of freedom equal to the dimension of $\theta$. The limiting $\chi^{2}$-distribution is central under $H_{0}$, and has the non-centrality parameter under $H_{1}$ equal to,

$\gamma=\Delta \theta^{T} M^{T} \Sigma^{-1} M \Delta \theta$

it is known ${ }^{[6]}$ that the detection power of $\tau$ (the probability of successful detection for given probability of false alarm) is an increasing function of $\gamma$.

In (28), the dependence on $\theta_{0}$ has been removed for simplicity. The only term which should be computed after data collection is residual $D_{N}$ in (24). The statistical properties of $\tau$ provide a theoretical guideline for the choice of a threshold $\lambda_{\varepsilon}$ found from a $\chi^{2}$-table, where $\varepsilon$ is the false alarm rate specified by the users. If $\tau$ is found to be larger than the threshold value, then a change in parameter is detected. Therefore, the fault detection decision is performed by the rule,

$\left\{\begin{array}{l}\tau \leq \lambda_{\varepsilon}, \text { under } H_{0} \\ \tau>\lambda_{\varepsilon}, \text { under } H_{1}\end{array}\right.$

Denoting the parameter changes as $\left[\begin{array}{lll}\Delta \theta_{1} & \cdots & \Delta \theta_{l}\end{array}\right]^{T}$, in case that only $\Delta \theta_{i}$ is no-zero and all the other $\Delta \theta_{j}(j \neq i)$ are zero, the sufficient condition for detectability of the fault can be derived as, $\left|\Delta \theta_{i}\right| \geq \alpha_{i}$

where, $\alpha_{i}=\frac{\lambda_{\varepsilon}}{\sqrt{F_{i i}}}$

and $F_{i i}$ indicates the $(i, i)$ element of the Fisher information matrix,

$F=M^{T} \Sigma^{-1} M$

which indicates that the magnitude of fault should be large enough to guarantee detectability. 
Fault isolation and isolatability: The problem of fault isolation poses a bigger challenge than fault detection. The fault isolation is performed only after the deliverance of a fault detection alarm. Once a change is detected from the model parameter, it may be necessary to isolate which or which set parameters have changed. The statistical approach to residual evaluation requires the knowledge of the statistical properties of the residuals. For this purpose, divide $\theta$ into,

$\theta=\left[\begin{array}{l}\theta_{a} \\ \theta_{b}\end{array}\right]$

with $l_{a}=\operatorname{dim}\left(\theta_{a}\right), \quad l_{b}=\operatorname{dim}\left(\theta_{b}\right)$, where $l_{a}+l_{b}=l$. Make the corresponding partition of $M$, $M=\left[\begin{array}{ll}M_{a} & M_{b}\end{array}\right]$

which corresponds to the partition of $\theta$, i.e. the column dimension of $M_{a}$ is the same as the row dimension of $\theta_{a}$ and the column dimension of $M_{b}$ is the same as the row dimension of $\theta_{b}$, so that $M \theta=M_{a} \theta_{a}+M_{b} \theta_{b}$.

The problem now is to detect changes in $\theta_{a}$, where the sizes $l_{a}$ and $l_{b}$ of the components are assumed to be fixed and known. The problem of fault isolation can be formulated as the following hypotheses test,

$$
H_{a 0}:\left(\Delta \theta_{a}=0, \Delta \theta_{b}=0\right), H_{a 1}:\left(\Delta \theta_{a} \neq 0, \Delta \theta_{b}=0\right)
$$

The sensitivity test $\tilde{\tau}_{a}$ for monitoring $\Delta \theta_{a}$ is a generalized log-likelihood ratio test defined as,

$$
\tilde{\tau}_{a}=\widetilde{r}_{a}^{T} F_{a a}^{-1} \widetilde{r}_{a}
$$

where,

$$
\widetilde{r}_{a}=M_{a}^{T} \Sigma^{-1} D_{N}
$$

is the partial score in $\theta_{a}$ and $F_{a a}=M_{a}^{T} \Sigma^{-1} M_{a}$ is the covariance matrix of $D_{N}$. Under both hypothesis $\left(H_{a 0}\right.$ and $\left.H_{a 1}\right), \tilde{\tau}_{a}$ is a $\chi^{2}$-test with $l_{a}$ degrees of freedom. This distribution is a central $\chi^{2}$ under the $H_{a 0}$ hypothesis, and a non-central $\chi^{2}$ under the $H_{a 1}$ hypothesis with non-centrality parameter, $\widetilde{\gamma}_{a}=\Delta \theta_{a}^{T} F_{a a} \Delta \theta_{a}$

The statistical properties of $\tilde{\tau}_{a}$ provide a theoretical guideline for the choice of a threshold based on the $\chi^{2}$-table and desired false isolation probability $\rho$. Let $\lambda_{\rho}$ be the chosen threshold, the sensitivity test based isolation of a particular fault (e.g. $\Delta \theta_{a}$ ) can be made according to the following comparison,

$\left\{\begin{array}{l}\tilde{\tau}_{a} \leq \lambda_{\rho}, \text { under } H_{a 0} \\ \tilde{\tau}_{a}>\lambda_{\rho}, \text { under } H_{a 1}\end{array}\right.$
In some practice cases, we need to isolate the change in every individual parameter, which can be called complete isolation, in other cases; we just need to isolate the changes in some particular parameter subsets, which can be called partial isolation. In complete isolation cases, the test should be attribute on every individual parameter, or in other word, $\Delta \theta_{a}$ is considered as a particular parameter. On other hand, in partial isolation cases, $\Delta \theta_{a}$ is considered as a vector, which indicates a particular subset of parameters.

For the min-max test, the parameters in $\theta_{b}$ are considered as nuisance and are statistically rejected. Let the Fisher information matrix according to,

$F=\left[\begin{array}{ll}F_{a a} & F_{a b} \\ F_{b a} & F_{b b}\end{array}\right]=\left[\begin{array}{ll}M_{a}^{T} \Sigma^{-1} M_{a} & M_{a}^{T} \Sigma^{-1} M_{b} \\ M_{b}^{T} \Sigma^{-1} M_{a} & M_{b}^{T} \Sigma^{-1} M_{b}\end{array}\right]$

according to the parameter partition, plays a key role in statistical detection. Its importance as an information measure is a central issue in the derivation of the criteria proposed in this paper.

Letting $\Delta \theta_{a}=\Delta \theta_{i}, \quad\left(i=1, \cdots, l_{a}\right), \quad$ a necessary condition for complete isolatability is that the incidence matrix $M$ has full column rank, and a sufficient condition for complete isolatability of the fault $\Delta \theta_{i} \neq 0$ can be derived as,

$\left|\Delta \theta_{i}\right| \geq \beta_{i}$

where,

$\beta_{i}=\frac{\lambda_{\sigma}}{\sqrt{F_{a}(i, i)}}$

and $F_{a}(i, i)$ indicates the $(i, i)$ element of the covariance matrix $F_{a}=F_{a a}-F_{a b} F_{b b}^{-1} F_{b a}$ of $\tau_{a}^{*}=\widetilde{r}_{a}-F_{a b} F_{b b}^{-1} \widetilde{r}_{b}$ where $\widetilde{r}_{b}=M_{b}^{T} \Sigma^{-1} D_{N}$ is the partial score in $\theta_{b}$.

In the case when $\Delta \theta_{a}=\Delta \theta$ (fault in all parameters), the sensitivity test $\tilde{\tau}_{a}$ can be derived as,

$\tilde{\tau}_{a}=D_{N}^{T} \Sigma^{-1} M F^{-1} M^{T} \Sigma^{-1} D_{N}$

and the sufficient condition for complete isolatability of the faults $\Delta \theta_{i} \neq 0$ is expressed as,

$$
\left|\Delta \theta_{i}\right| \geq \frac{\lambda_{\sigma}}{\sqrt{F(i, i)}}
$$

\section{MAGLEV SYSTEM}

Process description: In this section, a design example will be presented to illustrate the design procedure of the proposed FDI methods. We introduced a MAGLEV system [11] to illustrate the validity of the proposed 
algorithm. The electromagnet-track configuration is illustrated on Fig.1.

At the equilibrium point $\left(i_{0}, z_{0}\right)$, to derive the model of the open-loop system, the parameters of the magnet at the equilibrium point need to be calculated. In addition, if the disturbance force is assumed to be zero, the open-loop transfer function of the MAGLEV system is given by,

$$
\frac{Z(s)}{V(s)}=\frac{-\left(k_{i} / m R\right)}{\left(1+T_{m} s\right)\left(s^{2}+\frac{k_{i}^{2}}{m R\left(1+T_{m} s\right)} s-\frac{k_{z}}{m}\right)}
$$

where $z(t)$ the air-gap, $v(t)$ the instantaneous voltage, $m$ the suspended mass, $R$ the total resistance of the circuit, $L$ the inductance of the magnet winding, $T_{m}=L / R$ the magnet winding electrical time constant, $k_{i}$ the slope of the force-current characteristics, and $k_{z}$ the slope of the force-distance characteristics.

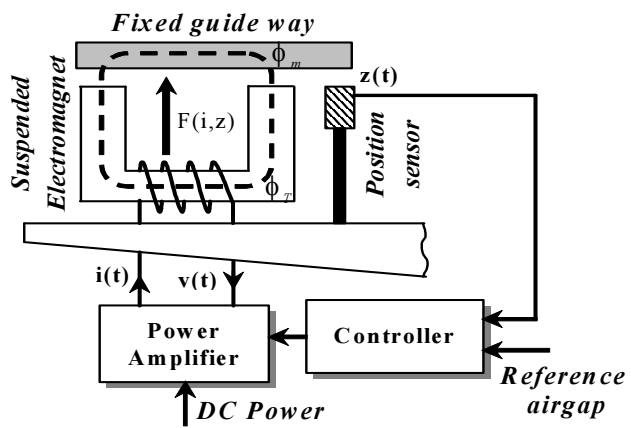

Fig.1: Experimental electromagnetic suspension system

The open-loop transfer function (46) indicates that the lag due to the magnet winding electrical time constant, $T_{m}$, may be separated from the suspension dynamics. If the power amplifier has a wide enough bandwidth, $T_{m}<<1$, then an approximate form of openloop characteristic equation is,

$$
\left(1+T_{m} s\right)\left(s^{2}+\frac{k_{i}^{2}}{m R} s-\frac{k_{z}}{m}\right)=0
$$

Finally, the open-loop transfer function of the MAGLEV system is expressed as,

$$
\frac{Z(s)}{V(s)}=\frac{-\left(k_{i} / m L_{0}\right)}{\left(s+\frac{1}{T_{m}}\right)\left(s^{2}+\frac{k_{i}^{2}}{m R} s-\frac{k_{z}}{m}\right)}
$$

We can represent the MAGLEV system by the following input-output model,

$\frac{d z^{2}(t)}{d t^{2}}+a_{1} \frac{d z(t)}{d t}+a_{0} z(t)=b_{0} v_{1}(t)$

where, $\frac{d v_{1}(t)}{d t}+\frac{1}{T_{m}} v_{1}(t)=v(t)$

and, $a_{1}=\frac{k_{i}^{2}}{m R}, a_{0}=-\frac{k_{z}}{m}, b_{0}=-\frac{k_{i}}{m L}$

Hence, the incidence matrix is depicted in the following table,

Table 1: Incidence matrix

\begin{tabular}{ccccc}
\hline & $R$ & $L$ & $k_{z}$ & $k_{i}$ \\
\hline$a_{1}$ & 1 & 0 & 0 & 1 \\
$a_{0}$ & 0 & 0 & 1 & 0 \\
$b_{0}$ & 0 & 1 & 0 & 1 \\
\hline
\end{tabular}

Due to the nonlinear force-distance and forcecurrent characteristics, MAGLEV system is unstable in open-loop feedback of at least position through a leadlag compensator is needed to obtain stability, though feedback of vertical acceleration is commonly incorporated to gain adequate control over suspension characteristics [11]. Therefore, the open-loop poles in (48) suggest that at least one zero is needed if the system is to be stabilized by using the classical compensation techniques. The control law derived by the compensator may be expressed as,

$v(t)=k_{p} c(t)+k_{a} \frac{d^{2} z(t)}{d t^{2}}$

where $k_{p}, k_{a}$ are the appropriate feedback gains, and $c(t)$ is the clearance measured with respect to the instantaneous guide-way height $h(t)$, Fig.2.

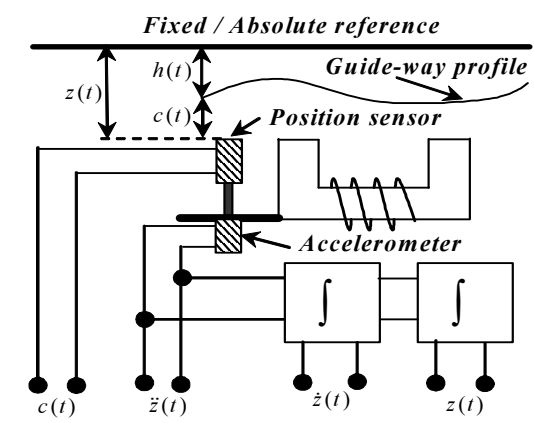

Fig.2: System configuration with an absolute reference

MAGLEV delta model: Using the delta operator (section 2), a discrete-time representation of the previous model (49) is given by,

$z(t)=[E(\delta)-A(\delta)] z_{F}(t)+B(\delta) v_{1 F}(t)$

where,

$A(\delta)=\delta^{2}+a_{1} \delta+a_{0}, \quad B(\delta)=b_{0}$

and, 


$$
\left\{\begin{array}{l}
z_{F}(t)=\frac{z(t)}{E(\delta)}, \quad v_{1 F}(t)=\frac{v_{1}(t)}{E(\delta)} \\
E(\delta)=\delta^{2}+e_{1} \delta+e_{0}
\end{array}\right.
$$

We can have the model in the following form,

$z(t)=\varphi_{F}^{T}(t) \theta$

where,

$\varphi_{F}^{T}(t)=\left[\delta z_{F}(t) \quad z_{F}(t) \quad v_{1 F}(t)\right]$

and,

$\theta=\left[\begin{array}{lll}\left(e_{1}-a_{1}\right) & \left(e_{0}-a_{0}\right) & b_{0}\end{array}\right]^{T}$

The regression vector $\varphi_{F}(t)$ can be generated by,

$\left\{\begin{array}{l}\delta \varphi_{y}(t)=\left[\begin{array}{cc}e_{1} & e_{0} \\ 1 & 0\end{array}\right] \varphi_{y}(t)+\left[\begin{array}{l}1 \\ 0\end{array}\right] z(t) \\ \delta \varphi_{u}(t)=e_{0} \varphi_{u}(t)+v_{1}(t)\end{array}\right.$

where,

$\varphi_{y}^{T}(t)=\left[\delta z_{F}(t) \quad z_{F}(t)\right], \quad \varphi_{u}^{T}(t)=\left[v_{1 F}(t)\right]$

\section{RESULTS AND DISCUSSIONS}

MAGLEV Model simulations: At the equilibrium point $(2 A, 1.5 \mathrm{~mm})$, the nominal values of the MAGLEV physical parameters are presented in table 2 .

Table 2: Physical parameters for the MAGLEV system

\begin{tabular}{ccccc}
\hline$m,(\mathrm{~kg})$ & $L,(m H)$ & $R,(\Omega)$ & $k_{z},(N / m)$ & $k_{i},(N / A)$ \\
\hline 3 & 33 & 7 & 58000 & 44 \\
\hline
\end{tabular}

Therefore, the nominal values of the model parameters are,

$$
a_{1}=92.2, a_{0}=-19333, b_{0}=-0.45
$$

A set of training data with a sampling rate $T=10 \mathrm{~ms}$ (20s correspond to $N=2000$ sample points) is first simulated when no fault occurs in the process. The nominal parameters of (58) are estimated by applying the HLS algorithm with forgetting factor (14) to the training data, where,$$
\hat{a}_{1}=91.8797, \hat{a}_{0}=-19238, \hat{b}_{0}=-0.4423
$$

A noise signal with standard deviation $\sigma=0.94$ corrupted output signal $z(t)$ simulated with the nominal parameter $\theta_{0}$ is depicted in Fig.3.
}

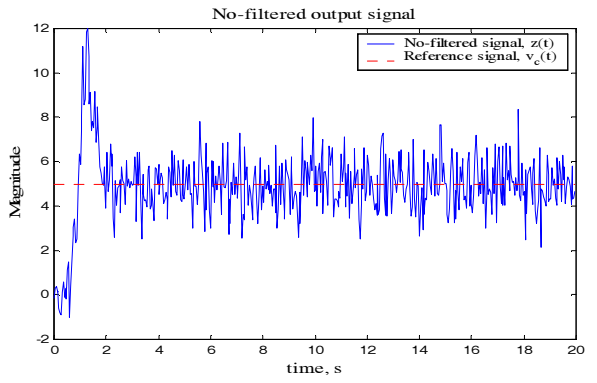

Fig.3: No-filtered output simulated with the nominal parameters

In order to let the reader appreciate the small parameter changes considered in our simulations, the low-pass filters can be applied to $z(t)$ to reduce noise and the effects of un-modeled dynamics. Many lowpass filtering techniques are available in the literature. Simple second-order Butterworth filters with cut-off frequencies at $0.1 \mathrm{~Hz}$ have been used here to process the output, Fig.4.

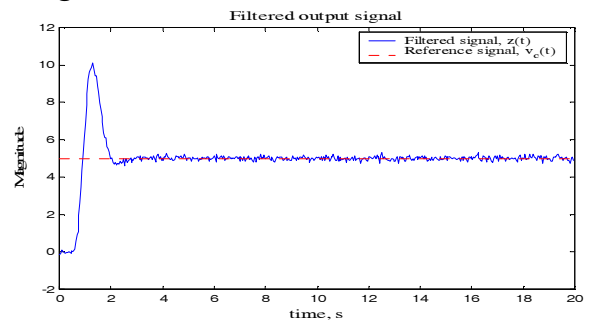

Fig.4: Filtered output simulated with the nominal parameters

MAGLEV faults simulated: Six cases are considered in the simulation:

1. Change in a single parameter:

- Case 1: Fault $F_{1}, 1 \%$ increase of $a_{1}:\left|\Delta a_{1}\right|=0.922$

- Case 2: Fault $F_{2}, 3 \%$ decrease of $a_{0}:\left|\Delta a_{0}\right|=580$

- Case 3: Fault $F_{3}, 1 \%$ decrease of $b_{0}$ : $\left|\Delta b_{0}\right|=0.0045$

2. Change in a pair of parameters:

- Case 4: Fault $F_{4}, 1 \%$ increase of $a_{1}$ and $3 \%$ decrease of $a_{0}$

- Case 5: Fault $F_{5}, 3 \%$ decrease of $a_{0}$ and $1 \%$ decrease of $b_{0}$

3. Change in a three parameters:

- Case 6: Fault $F_{6}, 1 \%$ increase of $a_{1}, 3 \%$ decrease of $a_{0}$ and $1 \%$ decrease of $b_{0}$

The considered model parameters values for the above cases are summarized in table 3. 
Table 3: Considered parameters values

\begin{tabular}{|c|c|c|c|c|c|c|}
\hline & $\begin{array}{l}\text { Case } 1 \\
\end{array}$ & Case 2 & $\begin{array}{l}\text { Case } 3 \\
\end{array}$ & Case 4 & Case 5 & Case 6 \\
\hline$a_{1}$ & 93.12 & 92.20 & 92.20 & 93.12 & 92.20 & 93.12 \\
\hline$a_{0}$ & -19333 & -19913 & -19333 & -19913 & -19913 & $19 \overline{913}$ \\
\hline$b_{0}$ & -0.45 & -0.45 & -0.4545 & -0.45 & -0.4545 & $\begin{array}{c}- \\
04545\end{array}$ \\
\hline
\end{tabular}

The question is: Can we detect any one of the possible faults with a small magnitude subject to measurement noises? Can we isolate the source of the faults?

Fault detection test: The global test (28) is applied to data simulated with the nominal model $\theta_{0}$, and to data simulated with modified parameters $\theta$. For each simulation, the sample size is $N=2000$. According to the $\chi^{2}$-table, applying the threshold $\lambda_{\varepsilon}=11.34$ (degrees of freedom, $l=3$ ) to this test corresponds, in theory, to the false alarm probability of $\varepsilon=0.01$. The results are summarized in tables 4-6.

Table 4: Fault detection test, $\sigma=0.1, N=2000$

\begin{tabular}{ccccccc}
\hline & Case 1 & Case 2 & Case 3 & Case 4 & Case 5 & Case 6 \\
\hline$\tau$ & 55.55 & 53.45 & 41.74 & 50.18 & 21.49 & 22.51 \\
$\gamma$ & 9.25 & 1.63 & 8.66 & 1.63 & 1.41 & 1.41 \\
\hline
\end{tabular}

Table 5: Fault detection test, $\sigma=0.4, N=2000$

\begin{tabular}{ccccccc}
\hline & Case 1 & Case 2 & Case 3 & Case 4 & Case 5 & Case 6 \\
\hline$\tau$ & 24.52 & 41.81 & 25.78 & 29.65 & 13.26 & 14.11 \\
$\gamma$ & 2.49 & 2.07 & 8.02 & 1.07 & 1.23 & 1.13 \\
\hline
\end{tabular}

Table 6: Fault detection test, $\sigma=0.4, N=3000$

\begin{tabular}{ccccccc}
\hline & Case 1 & Case 2 & Case 3 & Case 4 & Case 5 & Case 6 \\
$\tau$ & 44.53 & 51.84 & 35.73 & 49.74 & 17.39 & 21.14 \\
$\gamma$ & 7.39 & 2.13 & 8.62 & 2.07 & 1.83 & 1.83 \\
\hline
\end{tabular}

Comments: It is observed from the above tables 4-5 that when fault occurs, the test $\tau$ is greater than $\lambda_{\varepsilon}$. For example in case 1 , the test $\tau$ is equal to 55.55 and 24.52 for $\sigma=0.1$ and $\sigma=0.4$, respectively (Tables 4, $5)$. Both tests are greater than the threshold $\lambda_{\varepsilon}=11.34$, then the fault $F_{1}$ is detected, but the global test when $\sigma=0.4$ is less than the global test when $\sigma=0.1$, due to the noise effect. From the same noise standard deviation of $\sigma=0.4$, the fault detection performance are improved by increasing the sample size from 2000 to 3000 , in which the test $\tau$ is equal to 44.53 and 24.52 when $N=3000$ and $N=2000$ respectively (Tables 5, 6). For the same procedure, all faults $F_{2}, F_{3}, F_{4}, F_{5}$, and $F_{6}$ are detected.
Fault detectability test: The sufficient conditions for fault detectability (32-33) results are shown in the following tables 7-9, which correspond to the standard deviation of measurement noise equal to 0.1 , and 0.4 , respectively.

Table 7: Fault detectability test, $\sigma=0.1, N=2000$

\begin{tabular}{ccccccc}
\hline & Case 1 & Case 2 & Case 3 & Case 4 & Case 5 & Case 6 \\
\hline$\alpha_{1}$ & 0.24 & 53.84 & 78.24 & 0.40 & 25.65 & 0.26 \\
$\alpha_{2}$ & 962.9 & 275.5 & 802.6 & 267.9 & 355.1 & 382.8 \\
$\alpha_{3}$ & 0.49 & 0.43 & 0.0033 & 0.41 & 0.0029 & 0.0021 \\
\hline
\end{tabular}

Table 8: Fault detectability test, $\sigma=0.4, N=2000$

\begin{tabular}{|c|c|c|c|c|c|c|}
\hline & Case 1 & Case 2 & Case 3 & Case 4 & Case 5 & Case 6 \\
\hline$\alpha_{1}$ & 0.74 & 17.02 & 23.59 & 0.67 & 16.91 & 0.32 \\
\hline$\alpha_{2}$ & 666.3 & 428.9 & 659.1 & 415.8 & 211.9 & 427.3 \\
\hline$\alpha_{3}$ & 0.24 & 0.19 & 0.0042 & 0.17 & 0.0041 & 0.0043 \\
\hline
\end{tabular}

Table 9: Fault detectability test, $\sigma=0.4, N=3000$

\begin{tabular}{ccccccc}
\hline & Case 1 & Case 2 & Case 3 & Case 4 & Case 5 & Case 6 \\
\hline$\alpha_{1}$ & 0.52 & 47.23 & 63.51 & 0.46 & 21.69 & 0.27 \\
$\alpha_{2}$ & 817.2 & 343.2 & 745.9 & 395.7 & 321.8 & 370.2 \\
$\alpha_{3}$ & 0.35 & 0.32 & 0.0035 & 0.36 & 0.0031 & 0.0029 \\
\hline
\end{tabular}

Comments: From the tables 7-8, we note that the sufficient condition for fault detectability is satisfied. For example, in case 1 , we have $\left(\alpha_{1}=0.24\right.$, $\left.\alpha_{2}=962.9, \alpha_{3}=0.49\right)$ and $\left(\alpha_{1}=0.74, \alpha_{2}=666.3\right.$, $\alpha_{3}=0.24$ ) for $\sigma=0.1$ and $\sigma=0.4$, respectively, where $\left|\Delta \theta_{1}\right|=0.922,\left|\Delta \theta_{2}\right|=0$ and $\left|\Delta \theta_{3}\right|=0$, we note that $\left|\Delta \theta_{1}\right|>\alpha_{1},\left|\Delta \theta_{2}\right|<\alpha_{2}$ and $\left|\Delta \theta_{3}\right|<\alpha_{3}$ then the fault detectability test for the model parameter $a_{1}$ is satisfied. Therefore, for all cases, the fault detectability test is satisfied, but the result is near perfect when the noise standard deviation increases. From the same noise standard deviation of $\sigma=0.4$, the fault detectability performance are improved by increasing the sample size from 2000 to 3000 , in which in the case 1 , $\left(\alpha_{1}=0.74, \alpha_{2}=666.3, \alpha_{3}=0.24\right)$ and $\left(\alpha_{1}=0.52\right.$, $\left.\alpha_{2}=817.2, \quad \alpha_{3}=0.35\right) \quad$ when $\quad N=2000 \quad$ and $N=3000$, respectively (Tables 8,9 ).

Fault isolation test: After the deliverance of a fault detection alarm, the test (37-38) is applied to isolate this fault. According to the $\chi^{2}$-table, applying the threshold $\lambda_{\mathrm{v}}=6.63, \lambda_{\rho}=9.21$, and $\lambda_{\rho}=11.34\left(l_{a}=1, l_{a}=2\right.$, 
$l_{a}=3$ respectively), corresponds, in theory, to the false alarm probability of $\rho=0.01$. The results are summarized in tables 10-11.

Comments: From the tables 10-11, we conclude that all faults detected are isolated.

- Case 1: The test $\tilde{\tau}_{a}$ is equal to 12.51 and 8.56 for $\sigma=0.1$ and $\sigma=0.4$, respectively, it is clear that $\tilde{\tau}_{a}$ is greater then the threshold $\left(\lambda_{\mathrm{v}}=6.63\right)$, then the fault in the model parameter $a_{1}$ is isolated but near perfect when the noise standard deviation increase. According to the incidence matrix, table 1 , we can conclude that the fault in the physical parameter $R$ is isolated.

Table 10: Fault isolation test, $\sigma=0.1, N=2000$

\begin{tabular}{ccccccc}
\hline & Case 1 & Case 2 & Case 3 & Case 4 & Case 5 & Case 6 \\
\hline$\tilde{\tau}_{a}$ & 12.51 & 10.84 & 11.72 & 17.08 & 14.94 & 22.51 \\
$\tilde{\gamma}_{a}$ & 4.72 & 2.70 & 3.23 & 4.33 & 3.63 & 2.63 \\
\hline
\end{tabular}

Table 11: Fault isolation test, $\sigma=0.4, N=2000$

\begin{tabular}{ccccccc}
\hline & Case 1 & Case 2 & Case 3 & Case 4 & Case 5 & Case 6 \\
\hline$\widetilde{\tau}_{a}$ & 8.56 & 7.16 & 7.28 & 11.18 & 10.32 & 14.11 \\
$\widetilde{\gamma}_{a}$ & 3.66 & 1.01 & 1.93 & 2.02 & 1.53 & 1.16 \\
\hline
\end{tabular}

For the same procedure, all faults are isolated, where:

- Case 2: The fault in the model parameter $a_{0}$ is isolated, and according to the incidence matrix, the fault in the physical parameter $k_{w}$ is isolated.

- Case 3: The fault in the model parameter $b_{0}$ is isolated, and according to the incidence matrix, the faults in the physical parameters $L$ and $k_{z}$ are isolated.

- Case 4: The fault in the model parameters $a_{1}$ and $a_{0}$ are isolated, and according to the incidence matrix, the faults in the physical parameters $R, k_{z}$ and $k_{i}$ are isolated.

- Case 5: The fault in the model parameter $a_{0}$ and $b_{0}$ are isolated, and according to the incidence matrix, the faults in the physical parameters $L$, $k_{z}$ and $k_{z}$ are isolated.

- Case 6: The fault in the model parameter $a_{1}, a_{0}$ and $b_{0}$ are isolated, and according to the incidence matrix the faults in all physical parameters are isolated.

Fault isolatability test: The sufficient conditions for fault isolatability (42-43) results are shown in the following tables 12-13.
Table 12: Fault isolatability test, $\sigma=0.1, N=2000$

\begin{tabular}{ccccccc}
\hline & Case 1 & Case 2 & Case 3 & Case 4 & Case 5 & Case 6 \\
\hline$\beta_{1}$ & 0.21 & - & - & 0.23 & - & 0.26 \\
$\beta_{2}$ & - & 100.8 & - & 154.5 & 228.8 & 382.8 \\
$\beta_{3}$ & - & - & 0.0024 & - & 0.0037 & 0.0021 \\
\hline
\end{tabular}

Table 13: Fault isolatability test, $\sigma=0.4, N=2000$

\begin{tabular}{ccccccc}
\hline & Case 1 & Case 2 & Case 3 & Case 4 & Case 5 & Case 6 \\
\hline$\beta_{1}$ & 0.64 & - & - & 0.52 & - & 0.32 \\
$\beta_{2}$ & - & 265.5 & - & 353.1 & 421.8 & 427.3 \\
$\beta_{3}$ & - & - & 0.0038 & - & 0.0041 & 0.0043 \\
\hline
\end{tabular}

Comments: The sufficient condition for fault isolatability $\left|\Delta \theta_{i}\right|$ is satisfied. For example, in case 1, $\beta_{1}=0.21$ for $\sigma=0.1$ and $\beta_{1}=0.64$ for $\sigma=0.4$, where $\left|\Delta \theta_{1}\right|=0.922$, we note that $\left|\Delta \theta_{1}\right|>\beta_{1}$ for both tests, then the condition test for fault isolatability for the model parameter $a_{1}$ is satisfied. Therefore, the condition test for fault isolatability for the physical parameter $R$ is satisfied.

\section{CONCLUSION}

A new FDI approach has been proposed. The combination between delta operator and statistical local approach offers advantages to generate and develop a new residual. This FDI technique has been developed for on-line abrupt change in physical parameters system. The results clearly show that the residual generation and evaluation method proposed in this study, offers a great potential for detection and diagnosis of physical parameters fault. The perspectives of this work are situated in the combination with other FDI techniques and the application on nonlinear systems.

\section{ACKNOWLEDGMENTS}

The authors would like to thank the anonymous referees for their valuable comments.

\section{REFERENCES}

1. Huang, B., 2001. Detection of Abrupt Changes of Total Lest Square Models and Application in Fault Detection. IEEE Transactions on Control Systems Technology, 9(2), 357-367.

2. Basseville, M. and I.V. Nikiforov, 1993. Detection of Abrupt Changes - Theory and Applications. Prentice-Hall, Inc., Simon \& Schuster Company, Englewood Cliffs, NJ, USA. 
3. Neuman, C., 1993. Properties of the Delta Operator Model of Dynamic Physical Systems. IEEE Transactions Systems Man and Cybernetics, 23(1), 296-301.

4. Middleton, R.H. and G.C. Goodwin, 1990. Digital Control and Estimation: A Unified Approach. Prentice Hall, NJ, USA.

5. Athamena, B. and H.A. Abbassi, 2003. Fault Detection and Isolation using Hybrid Parameter Estimation and Fuzzy Logic Residual Evaluation. International Journal of Computing and Informatics, 27(1), 29-37.

6. Athamena, B. and H.A. Abbassi, 2000. Fault Detection and Isolation using a Statistical Method and Hybrid Parameter Estimation. IEEE Sixth International Conference on Methods and Models in Automation and Robotics, Poland, 28-31.

7. Basseville, M., A. Benveniste, M. Goursat and L. Mevel, 2007. Subspace-Based Algorithms for Structural Identification, Damage Detection, and Sensor Data Fusion. Journal on Advances in Signal Processing, 1-13.
8. Basseville, M. and I.V. Nikiforov, 2005. Handling Nuisance Parameters in Systems Monitoring. Proceedings of the 44th IEEE Conference on Decision and Control, and the European Control Conference, Seville, Spain, 3832-3837.

9. Basseville, M., 1998. On-Board Component Fault Detection and Isolation using the Statistical Local Approach. Automatica, 34(11), 1391-1415.

10. Evsukoff, A., J. Montmain and S. Gentil, 1997. Dynamic Model and Causal Knowledge-Based Fault Detection and Isolation. IFAC Symposium on Fault Detection, Supervision and Safety Technical Process, Hull, UK, 699-704.

11. Sinha, P.K., 1987. Electromagnetic Suspension: Dynamics and Control. Peter Peregrinus on behalf of the Institution of Electrical Engineers, London, UK. 